\title{
Representing a nonlinear state space system as a set of higher-order differential equations in the inputs and outputs
}

\author{
A.J. van der SCHAFT \\ Dept. of Applied Mathematics, University of Twente, P.O. Box 217, 7500 AE Enschede, The Netherlands
}

Received 18 March 1988

Revised 3 October 1988

Abstract: An algorithm is presented for transforming a nonlinear state space system into a threefold set of equations; the first subset describing the dynamics of the unobservable part of the system, the second subset representing the remaining state variables as functions of inputs and outputs and their derivatives, and the last subset defining the external behaviour of the system.

AMS Subject Classifications: 93B17, 93B20.

Keywords: Nonlinear control system; equivalence transformation; observability; external differential system.

\section{Introduction}

This paper is concerned with the relation between the representation of a smooth nonlinear input-output system as a system in state space form on the one hand, and as a system given by a set of nonlinear implicit higher-order differential equations in the inputs and outputs, shortly external differential system, at the other hand. In a previous paper [11], see also [12], we investigated the conditions under which an external differential system can be represented (realized) as a state space system (and somewhat surprisingly it was found out these conditions are generically not met, see also Freedman \& Willems [6]). Conversely, in the present paper we give a simple algorithm to convert locally, avoiding singularities, a nonlinear state space system into an external differential system. The idea underlying this algorithm is simple (and has been used for different purposes already by other authors, see e.g. Respondek \& Nijmeijer [10]): differentiate the outputs in order to solve for a part of $\dot{x}$, the time derivative of the state, and differentiate once more to solve for a larger part of $\dot{x}$, and so on. However to our knowledge such an algorithm has never been exposed systematically before. Of course the idea of representing a system in state space form by an external differential system is very much implicit in some recent papers by Fliess (for example $[4,5]$ ), but an algorithm is not given there. Also our work is in a smooth $\left(C^{\infty}\right)_{-}$context, avoiding singularities, while Fliess emphasizes a differential algebraic approach. The present paper was very much motivated by the theory of equivalence transformations for linear systems, see especially Schumacher [14] and Willems [16,17]. Also we would like to draw attention to some very recent papers by Crouch \& Lamnabhi [2] and Sontag [15] about the relation between state space systems and external differential systems especially in the bilinear case.

An important consequence of the algorithm presented is that we not only obtain the equations describing the external differential system, but also a set of equations involving the inputs and outputs and their (higher-order) derivatives and the state variables (and not their derivatives). From these last equations we can solve for the state as function of the inputs and outputs and their derivatives if and only if the system is observable in some well-defined sense. This can be seen as the nonlinear extension of one of the classical interpretations of observability for a linear system. 
It came to our attention that recently also Conte, Moog \& Perdon [1] and Respondek [9] independently have proved that modulo singularities a smooth state space system can be transformed into an external differential system. Furthermore during the Nonlinear Control Conference in Nantes, June 1988, Glad [7] and Diop [3] have presented algorithms to convert polynomial state space systems into globally defined external differential systems, using tools from differential algebra (cf. Fliess [4,5]).

\section{The algorithm}

Consider a general smooth (i.e. $C^{\infty}$ ) nonlinear system

$$
\begin{aligned}
& \dot{x}=f(x, u), \\
& y=h(x, u) .
\end{aligned}
$$

Since our analysis will be mainly local we assume for simplicity that $x \in \mathbb{R}^{n}, u \in \mathbb{R}^{m}, y \in \mathbb{R}^{p}$. Our starting point is to rewrite (1) as a set of implicit equations

$$
\begin{aligned}
& P_{i}(x, \dot{x}, u)=\dot{x}_{i}-f_{i}(x, u)=0, \quad i=1, \ldots, n, \\
& P_{i}(x, u, y)=y_{i-n}-h_{i-n}(x, u)=0, \quad i=n+1, \ldots, n+p,
\end{aligned}
$$

in the indeterminates $x \in \mathbb{R}^{n}, \dot{x} \in \mathbb{R}^{n}, u \in \mathbb{R}^{m}, y \in \mathbb{R}^{p}$.

For a general (higher-order) differential equation

$$
R\left(w, \dot{w}, \ldots, w^{(k)}\right)=0, \quad w \in \mathbb{R}^{q},
$$

with $w^{(j)}$ the $j$-th derivative, the prolonged equation, or prolongation, is defined as

$$
\begin{aligned}
0 & =\dot{R}\left(w(t), \dot{w}(t), \ldots, w^{(k+1)}(t)\right):=\frac{\partial R}{\partial w} \dot{w}(t)+\frac{\partial R}{\partial \dot{w}} \ddot{w}(t)+\cdots+\frac{\partial R}{\partial w^{(k)}} w^{(k+1)}(t) \\
& =\frac{\mathrm{d}}{\mathrm{d} t} R\left(w(t), \dot{w}(t), \ldots, w^{(k)}(t)\right) .
\end{aligned}
$$

It is clear that if $w(t), t \in(a, b)$, is a solution of (3) then it is also a solution of (4). In the present context we consider the prolongations of the last $p$ equations in (2). Explicitly

$$
\dot{P}_{i}(x, \dot{x}, u, \dot{u}, y, \dot{y})=\dot{y}_{i-n}-\sum_{j=1}^{n} \frac{\partial h_{i-n}}{\partial x_{j}} \dot{x}_{j}-\sum_{l=1}^{m} \frac{\partial h_{i-n}}{\partial u_{l}} \dot{u}_{l}=0, \quad i=n+1, \ldots, n+p .
$$

Let us introduce some further terminology, cf. [11], borrowed from the theory of linear higher-order differential equations, see also $[14,16,17]$. The degree $\sigma$ of $(3)$ is defined as the highest time-derivative appearing non-trivially in (3); i.e. $\sigma$ is the largest integer $(\leq k)$ such that

$$
\frac{\partial R}{\partial w^{(\sigma)}}\left(w, \dot{w}, \ldots, w^{(k)}\right) \neq 0 \text { for some }\left(w, \dot{w}, \ldots, w^{(k)}\right) .
$$

If $\sigma$ is not defined (i.e. $R\left(w, \ldots, w^{(k)}\right)$ is constant), then set $\sigma=0$. It is easy to see that if $\sigma$ is the degree of (3) then

$$
\frac{\partial \dot{R}}{\partial w^{(\sigma+1)}}\left(w, \dot{w}, \ldots, w^{(k+1)}\right)=\frac{\partial R}{\partial w^{(\sigma)}}\left(w, \dot{w}, \ldots, w^{(k)}\right)
$$

and the degree of $\dot{R}$ equals $\sigma+1$.

Now consider a set of general higher-order equations

$$
R_{i}\left(w, \dot{w}, \ldots, w^{(k)}\right)=0, \quad i=1, \ldots, l .
$$


For any $i \in\{1,2, \ldots, l\}$ let $\sigma_{i}$ be the degree of $R_{i}$. The leading row coefficient matrix of (8) is defined as

$$
\left[\frac{\partial R_{i}}{\partial w_{j}^{\left(\boldsymbol{o}_{i}\right)}}\left(w, \dot{w}, \ldots, w^{(k)}\right)\right]_{\substack{i=1, \ldots, l \\ j=1, \ldots, q}} .
$$

Let us specialize this to equations (2), which will be regarded as equations in $x, \dot{x}$ with parameters $u, y$. It is clear that the degrees with respect to $x$ of the first $n$ equations in (2) are all one, while the degrees (w.r.t. $x$ ) of the last $p$ equations are all zero. Furthermore the leading row coefficient matrix (w.r.t. $x$ ) equals

$$
\left[\left[\frac{\partial P_{i}}{\partial x_{j}}(x, u, y)\right]_{\substack{i=n+1, \ldots, n+p \\ j=1 \ldots, \ldots, n}}^{I_{n}}\right]
$$

and clearly has rank $n$ everywhere. Furthermore (see (7))

$$
\frac{\partial \dot{P}_{i}}{\partial \dot{x}_{j}}(x, \dot{x}, u, \dot{u}, y, \dot{y})=\frac{\partial P_{i}}{\partial x_{j}}(x, u)=\frac{\partial h_{i-n}}{\partial x_{j}}(x, u), \quad i=n+1, \ldots, n+p ; j=1, \ldots, n .
$$

Now let $(\tilde{x}(t), \tilde{u}(t), \tilde{y}(t)), t \in(-\varepsilon, \varepsilon), \varepsilon>0$, be a solution of (1), yielding a solution point

$$
(\bar{x}, \bar{u}, \bar{y}):=\left(\tilde{x}(0), \dot{\tilde{x}}(0), \ldots, \tilde{x}^{(n)}(0), \tilde{u}(0), \ldots, \bar{u}^{(n)}(0), \tilde{y}(0), \ldots, \tilde{y}^{(n)}(0)\right)
$$

of (2). Around such a point $(\bar{x}, \bar{u}, \bar{y})$ we will set up an algorithm by which we increase in every step the number of equations in (2) which has degree 0.

\section{Algorithm.}

1-st step: Assume that

$$
\operatorname{rank}\left[\frac{\partial P_{i}}{\partial x_{j}}(x, u, y)\right]_{\substack{i=n+1, \ldots, n+p \\ j=1, \ldots, n}}=s_{1} \quad \text { around }(\bar{x}, \bar{u}, \bar{y}) .
$$

Denote $p_{1}:=s_{1}-s_{0}$, with $s_{0}=0$. If $p_{1}=0$ the algorithm terminates. If $p_{1}>0$ we proceed as follows. By (10) and (13) it follows that we can reorder the equations $P_{1}, \ldots, P_{n}$ and separately the equations $P_{n+1}, \ldots, P_{n+p}$ in such a way that

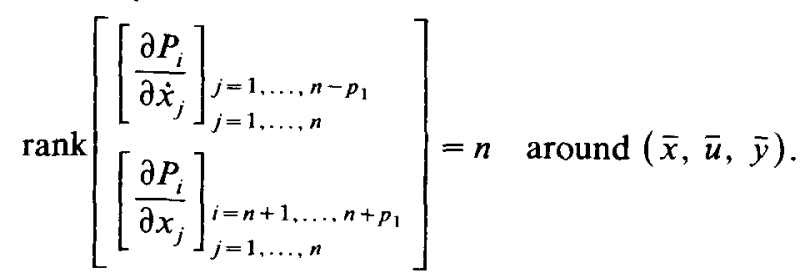

Furthermore we reorder the variables $x_{1}, \ldots, x_{n}$ in the same way as we did for $P_{1}, \ldots, P_{n}$, so that still $P_{i}(x, \dot{x}, u)=\dot{x}_{i}-f_{i}(x, u), i=1, \ldots, n$, and hence $\partial P_{i} / \partial \dot{x}_{j}=\delta_{i j}, i=1, \ldots, n-p_{1} ; j=1, \ldots, n$. Then clearly

$$
\operatorname{rank}\left[\frac{\partial P_{i}}{\partial x_{j}}\right]_{\substack{i=n+1, \ldots, n+p_{1} \\ j=n-p_{1}+1, \ldots . n}}=p_{1} \quad \text { around }(\bar{x}, \bar{u}, \bar{y}) .
$$

Now consider instead of (2) the set of equations

$$
\begin{aligned}
& P_{i}(x, \dot{x}, u)=\dot{x}_{i}-f_{i}(x, u)=0, \quad i=1, \ldots, n-p_{1}, \\
& \dot{P}_{i}(x, f(x, u), u, \dot{u}, y, \dot{y})=0, \quad i=n+1, \ldots, n+p_{1}, \\
& P_{i}(x, u, y)=0, \quad i=n+1, \ldots, n+p .
\end{aligned}
$$


It follows that the system of equations (16) is equivalent to (2) in the sense that a time-function $(x(t), u(t), y(t))$ close to $(\tilde{x}(t), \tilde{u}(t), \tilde{y}(t))$ is a solution of (2) if and only if it is a solution of (16). Indeed let $(x(t), u(t), y(t)$ be a solution curve of (2), then it is also a solution curve of the prolonged equations $\dot{P}_{n+1}=\cdots=\dot{P}_{n+p_{1}}=0$ and since $(2 \mathrm{a})$ is satisfied we may substitute in these equations $\dot{x}=f(x, u)$ to obtain (16b). Conversely, let $(x(t), u(t), y(t))$ be a solution curve of (16), then by (16c) it is also a solution curve of the prolonged equations $\dot{P}_{n+1}=\cdots=\dot{P}_{n+p_{1}}=0$ and hence, substituting also (16a),

$$
\sum_{j=1}^{n-p_{1}} \frac{\partial P_{i}}{\partial x_{j}} f_{j}(x, u)+\sum_{k=n-p_{1}+1}^{n} \frac{\partial P_{i}}{\partial x_{k}} \dot{x}_{k}+\sum_{s=1}^{p} \frac{\partial P_{i}}{\partial y_{s}} \dot{y}_{s}+\sum_{r=1}^{m} \frac{\partial P_{i}}{\partial u_{r}} \dot{u}_{r}=0, \quad i=n+1, \ldots n+p_{1} .
$$

On the other hand $(x(t), u(t), y(t))$ satisfies $(16 \mathrm{~b})$, i.e.

$$
\sum_{j=1}^{n-p_{1}} \frac{\partial P_{i}}{\partial x_{j}} f_{j}(x, u)+\sum_{k=n-p_{1}+1}^{n} \frac{\partial P_{i}}{\partial x_{k}} f_{k}(x, u)+\sum_{s=1}^{p} \frac{\partial P_{i}}{\partial y_{s}} \dot{y}_{s}+\sum_{r=1}^{m} \frac{\partial P_{i}}{\partial u_{r}} \dot{u}_{r}=0, \quad i=n+1, \ldots, n+p_{1} .
$$

By comparing (17) and (18), and using (15) it follows that $\dot{x}_{k}=f_{k}(x, u), k=n-p_{1}+1, \ldots, n$, so that $(x(t), u(t), y(t))$ satisfies $(2 a)$.

Finally rename the equations (16b) by setting

$$
P_{i}(x, u, \dot{u}, y, \dot{y}):=\dot{P}_{i+p_{1}}(x, f(x, u), u, \dot{u}, y, \dot{y}), \quad i=n-p_{1}+1, \ldots, n .
$$

Denote $n_{1}:=n$ and $n_{2}:=n_{1}-p_{1}$, then (16) is rewritten as

$$
\begin{aligned}
& P_{i}(x, \dot{x}, u)=\dot{x}_{i}-f_{i}(x, u)=0, \quad i=1, \ldots, n_{2}, \\
& P_{i}(x, u, \dot{u}, y, \dot{y})=0, \quad i=n_{2}+1, \ldots, n+p .
\end{aligned}
$$

As a result of the first step we have transformed (2) into an equivalent system (20) which has $p$, more equations with degree 0 . It is easily checked that the leading row coefficient matrix of (20) has rank $n$, as was the case for the original system (2).

$k$-th step: At the $k$-th step we start with a system of equations

$$
\begin{aligned}
& P_{i}(x, \dot{x}, u)=\dot{x}_{i}-f_{i}(x, u)=0 \quad i=1, \ldots, n_{k}, \\
& P_{i}\left(x, u, \dot{u}, \ldots, u^{(k-1)}, y, \ldots, y^{(k-1)}\right)=0, \quad i=n_{k}+1, \ldots, n+p,
\end{aligned}
$$

for which the leading row coefficient matrix has rank $n$. Assume that

$$
\operatorname{rank}\left[\frac{\partial P_{i}}{\partial x_{j}}\right]_{\substack{i=n_{k}+1, \ldots . n+p \\ j=1, \ldots, n}}=s_{k} \text { around }(\bar{x}, \bar{u}, \bar{y}) \text {. }
$$

Denote $p_{k}=s_{k}-s_{k-1}$. If $p_{k}=0$ the algorithm terminates. If $p_{k}>0$ we proceed as follows. By (7),

$$
\left[\frac{\partial P_{i}}{\partial x_{j}}\right]_{\substack{i=n_{k}+1 \ldots ., n+p \\ j=1, \ldots, n}}\left[\frac{\partial \dot{P}_{i}}{\partial \dot{x}_{j}}\right]_{\substack{i=n_{k}+1 \ldots \ldots n+p \\ j=1, \ldots, n}} .
$$

Furthermore in the $(k-1)$-th step we assumed that

$$
\operatorname{rank}\left[\frac{\partial P_{i}}{\partial x_{j}}\right]_{\substack{i=n_{k-1}+1, \ldots, n+p \\ j=1 \ldots \ldots n}}=\operatorname{rank}\left[\frac{\partial \dot{P}_{i}}{\partial \dot{x}_{j}}\right]_{\substack{i=n_{k-1}+1, \ldots, n+p \\ j=1, \ldots, n}}=s_{k-1}
$$

around $(\bar{x}, \bar{u}, \bar{y})$. Now consider the prolongations of the $p_{k-1}$ new equations obtained in the $(k-1)$-th step

$$
\dot{P}_{i}\left(x, \dot{x}, u, \ldots, u^{(k)}, y, \ldots, y^{(k)}\right)=0, \quad i=n_{k}+1, \ldots, n_{k-1} .
$$


By (24) it follows that there are functions $\alpha_{i l}\left(x, u, \ldots, u^{(k-1)}, y, \ldots, y^{(k-1)}\right), i=n_{k}+1, \ldots, n_{k-1}, l=$ $n_{k-1}+1, \ldots, n+p$, such that if we define the following modifications of equations (25):

$$
\begin{aligned}
S_{i}\left(x, \dot{x}, u, \ldots, u^{(k)}, y, \ldots, y^{(k)}\right):= & \dot{P}_{i}\left(x, \dot{x}, u, \ldots, u^{(k)}, y, \ldots, y^{(k)}\right) \\
& +\sum_{l=n_{k-1}+1}^{n+p} \alpha_{i l}\left(x, u, \ldots, u^{(k-1)}, y, \ldots, y^{(k-1)}\right) \\
& \cdot \dot{P}_{i}\left(x, \dot{x}, u, \ldots, u^{(k)}, y, \ldots, y^{(k)}\right), \\
i=n_{k}+1, \ldots, n_{k-1}, &
\end{aligned}
$$

then

$$
\frac{\partial S_{i}}{\partial \dot{x}_{j}}=0, \quad i=n_{k}+1, \ldots, n_{k-1} ; j=n_{k}+1, \ldots, n .
$$

By (22), (23) we can now reorder $S_{n_{k}+1}, \ldots, S_{n_{k-1}}$ in such a way that

$$
\operatorname{rank}\left[\frac{\partial S_{i}}{\partial \dot{x}_{j}}\right]_{\substack{i=n_{k}+1, \ldots, n_{k}+p_{k} \\ j=1, \ldots, n_{k}}}=p_{k} \quad \text { around }(\bar{x}, \bar{u}, \bar{y}) \text {. }
$$

It follows that we can permute the equations $P_{1}, \ldots, P_{n_{k}}$ such that

$$
\operatorname{rank}\left[\begin{array}{l}
{\left[\frac{\partial P_{i}}{\partial \dot{x}_{j}}\right]_{\substack{i=1, \ldots, n_{k}-p_{k} \\
j=1, \ldots, n_{k}}}} \\
{\left[\frac{\partial S_{i}}{\partial \dot{x}_{j}}\right]_{\substack{i=n_{k}+1, \ldots, n_{k}+p_{k} \\
j=1, \ldots, n_{k}}}}
\end{array}\right]=n_{k} \quad \text { around }(\bar{x}, \bar{u}, \bar{y}) \text {. }
$$

Furthermore, we reorder the variables $x_{1}, \ldots, x_{n_{k}}$ in the same way as we $\operatorname{did}$ for $P_{1}, \ldots, P_{n_{k}}$, so that still $P_{i}(x, \dot{x}, u)=\dot{x}_{i}-f_{i}(x, u), i=1, \ldots, n_{k}$.

Now consider instead of (21) the set of equations

$$
\begin{aligned}
& P_{i}(x, \dot{x}, u)=\dot{x}_{i}-f_{i}(x, u)=0, \quad i=1, \ldots, n_{k}-p_{k}, \\
& S_{i}\left(x, f(x, u), u, \dot{u}, \ldots, u^{(k)}, y, \ldots, y^{(k)}\right)=0, \quad i=n_{k}+1, \ldots, n_{k}+p_{k}, \\
& P_{i}\left(x, u, \dot{u}, \ldots, u^{(k-1)}, y, \ldots, y^{(k-1)}\right)=0, \quad i=n_{k}+1, \ldots, n+p .
\end{aligned}
$$

It follows again that the system of equations (30) is equivalent to (21):

Lemma 1. Around $(\bar{x}, \bar{u}, \bar{y})$ the set of solution curves $(x(t), u(t), y(t))$ of $(30)$ is equal to the set of solution curves of (21).

Proof. It is easily seen that any solution curve of (21) is a solution curve of (30). Conversely let $(x(t), u(t), y(t))$ be a solution curve of $(30)$ close to $(\tilde{x}(t), \tilde{u}(t), \tilde{y}(t))$. In order that it is also a solution curve of $(21)$ we only have to prove that it is a solution curve of

$$
P_{i}(x, \dot{x}, u)=\dot{x}_{i}-f_{i}(x, u)=0, \quad i=n_{k}-p_{k}+1, \ldots, n_{k} .
$$

Clearly $(x(t), u(t), y(t))$ is a solution curve of

$$
S_{i}\left(x, \dot{x}, u, \ldots, u^{(k)}, y, \ldots, y^{(k)}\right)=0, \quad i=n_{k}+1, \ldots, n_{k}+p_{k} .
$$

Therefore by (30b) and the fact that $S_{i}$ is linear in $\dot{x},(x(t), u(t), y(t))$ satisfies

$$
\sum_{j=1}^{n} \frac{\partial S_{i}}{\partial \dot{x}_{j}} \dot{x}_{j}=\sum_{j=1}^{n} \frac{\partial S_{i}}{\partial \dot{x}_{j}} f_{j}(x, u), \quad i=n_{k}+1, \ldots, n_{k}+p_{k} .
$$


However by (27) and (30a) equations (32) reduce to

$$
\sum_{j=n_{k}-p_{k}+1}^{n_{k}} \frac{\partial S_{i}}{\partial \dot{x}_{j}} \dot{x}_{j}=\sum_{j=n_{k}-p_{k}+1}^{n_{k}} \frac{\partial S_{i}}{\partial \dot{x}_{j}} f_{j}(x, u), \quad i=n_{k}+1, \ldots n_{k}+p_{k} .
$$

Furthermore by (28) and (29),

$$
\operatorname{rank}\left[\frac{\partial S_{i}}{\partial \dot{x}_{j}}\right]_{\substack{i=n_{k}+1 \ldots . . n_{k}+p_{k} \\ j=n_{k}-p_{k}+1 \ldots \ldots n_{k}}}=p_{k},
$$

and hence (33) implies (31), as required.

Finally rename equations (30b) by setting

$$
\begin{aligned}
& P_{i}\left(x, u, \ldots, u^{(k)}, y, \ldots, y^{(k)}\right):=\dot{P}_{i+p_{k}}\left(x, f(x, u), u, \ldots, u^{(k)}, y, \ldots, y^{(k)}\right), \\
& i=n_{k}-p_{k}+1, \ldots, n_{k} .
\end{aligned}
$$

Denote $n_{k+1}:=n_{k}-p_{k}$; then (30) is rewritten as

$$
\begin{aligned}
& P_{i}(x, \dot{x}, u)=\dot{x}_{i}-f_{i}(x, u)=0, \quad i=1, \ldots, n_{k+1}, \\
& P_{i}\left(x, u, \ldots, u^{(k)}, y, \ldots, y^{(k)}\right)=0, \quad i=n_{k+1}+1, \ldots, n+p,
\end{aligned}
$$

which is a system with leading row coefficient matrix of $\operatorname{rank} n$, and having $p_{k}$ more equations of degree 0 than (21).

If at every step in the above algorithm the constant rank assumption (22) is met in a neighborhood of $(\bar{x}, \bar{u}, \bar{y})$, then we call $(\bar{x}, \bar{u}, \bar{y})$ a regular point for the algorithm.

It is clear that for a regular point the algorithm terminates after a finite number, say $k^{*}$, of steps in the sense that $p_{k^{*}+1}=0$. Moreover $k^{*} \leq n$. Denote $\bar{n}=n_{k^{*}+1}$; then we end up with a system

$$
\begin{aligned}
& \dot{x}_{i}-f_{i}(x, u)=0, \quad i=1, \ldots, \bar{n}, \\
& P_{i}\left(x, u, \ldots, u^{\left(k^{*}\right)}, y, \ldots, y^{\left(k^{*}\right)}\right)=0, \quad i=\bar{n}+1, \ldots n+p .
\end{aligned}
$$

Let us first consider the case $\bar{n}=0$. Recall that at the $k^{*}$-th step we assumed that

$$
\operatorname{rank}\left[\frac{\partial P_{i}}{\partial x_{j}}\right]_{\substack{i=n_{k} *+1 \ldots, n+p \\ j=1 \ldots, n}}=s_{k^{*}} \text { around }(\bar{x}, \bar{u}, \bar{y}) .
$$

Comparing the recursive definitions of $n_{k}$ and $p_{k}$,

$$
\begin{aligned}
& n_{k+1}=n_{k}-p_{k}, \quad n_{1}=n, \quad k \geq 1, \\
& p_{k+1}=s_{k+1}-s_{k}, \quad s_{0}=0, \quad k \geq 0,
\end{aligned}
$$

we immediately get $n_{k}=n-s_{k-1}, k \geq 1$. Hence $\bar{n}=n_{k^{*}+1}=n-s_{k^{*}}$, and in case $\bar{n}=0$ we have $s_{k^{*}}=n$. Now reorder the equations $P_{1}, \ldots, P_{n+p}$ of (35) for $\bar{n}=0$ in such a way that

$$
\operatorname{rank}\left[\frac{\partial P_{i}}{\partial x_{j}}\right]_{\substack{i=1 \ldots \ldots n \\ j=1 \ldots \ldots n}}=n\left(=s_{k^{*}}\right)
$$

where by (36) the equations $P_{1}, \ldots, P_{n}$ can be taken from the set $P_{i}, i=n_{k^{*}}+1, \ldots, n+p$. Then by the implicit function theorem we can locally solve from

$$
P_{i}\left(x, u, \dot{u}, \ldots, u^{\left(k^{*}-1\right)}, y, \dot{y}, \ldots, y^{\left(k^{*}-1\right)}\right)=0, \quad i=1, \ldots, n,
$$


for the variables $x_{1}, \ldots, x_{n}$, i.e. around $(\bar{x}, \bar{u}, \bar{y})$,

$$
x_{i}=\psi_{i}\left(u, \dot{u}, \ldots, u^{\left(k^{*}-1\right)}, y, \dot{y}, \ldots, y^{\left(k^{*}-1\right)}\right), \quad i=1, \ldots, n .
$$

Substitution of (40a) in the remaining equations $P_{n+1}, \ldots, P_{n+p}$ yields equations of the form

$$
P_{i}^{\mathrm{e}}\left(u, \dot{u}, \ldots, u^{\left(k^{*}\right)}, y, \dot{y}, \ldots, y^{\left(k^{*}\right)}\right)=0, \quad i=1, \ldots, p .
$$

We summarize this in the following theorem:

Theorem 2. Consider the system (1), and let $(\bar{x}, \bar{u}, \bar{y})$ be a regular point for the algorithm. Suppose that $\bar{n}=0$. Then (1) can be transformed around $(\bar{x}, \bar{u}, \bar{y})$ into the equations (40). The first equations (40a) express the state $x$ as function of $u$ and $y$ and their time-derivatives, while the last equations $(40 \mathrm{~b})$ describe the external behaviour of the system.

We shall now show that the assumption $\bar{n}=0$ exactly corresponds to the assumption of a notion of observability for (1), which is defined in the following way (see also [13]).

Definition 3. Consider a general smooth nonlinear system (1). Its extended system is given as

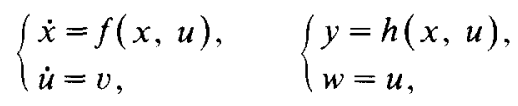

with inputs $v$, state $(x, u)$, outputs $(y, w)$ and output map $h^{\mathrm{e}}(x, u)=(h(x, u), u)$. The unobservability distribution $O$ of (41) is defined in the usual way (cf. [8]) as the largest distribution $D$ on $\mathbb{R}^{n} \times \mathbb{R}^{m}$ such that

$$
\begin{aligned}
& D \subset \text { ker } \mathrm{d} h^{\mathrm{e}}, \\
& {\left[f(x, u) \frac{\partial}{\partial x}, D\right] \subset D,} \\
& {\left[\frac{\partial}{\partial u_{j}}, D\right] \subset D, \quad j=1, \ldots, m .}
\end{aligned}
$$

From (42a, c) it follows [13] that $O$ may only have components in $\partial / \partial x$-directions and does not depend on $u$. Hence $O$ can be identified with a distribution $\bar{O}$ on $\mathbb{R}^{n}$ which by $(42 \mathrm{a}, \mathrm{b})$ satisfies

$$
[f(\cdot, u), \bar{O}] \subset \bar{O}, \quad \bar{O}(x) \subset \operatorname{ker}_{x} h(x, u), \quad \forall u \in \mathbb{R}^{m} \text {. }
$$

If $\operatorname{dim} \bar{O}=0$ then (1) is called locally observable. If $\operatorname{dim} \bar{O}(x)$ is constant, say $\bar{n}>0$, then by Frobenius' theorem we may choose coordinates $x=\left(x^{1}, x^{2}\right), \operatorname{dim} x^{1}=\bar{n}$, such that $\bar{O}=\operatorname{span}\left\{\partial / \partial x^{1}\right\}$, and by (43) the system takes the form

$$
\dot{x}^{1}=f^{1}\left(x^{1}, x^{2}, u\right), \quad \dot{x}^{2}=f^{2}\left(x^{2}, u\right), \quad y=h\left(x^{2}, u\right) .
$$

The variables $x^{1}$ are called the unobservable part of (44).

Remark. For an affine nonlinear system (1), i.e. $f(x, u)=f(x)+\sum_{j=1}^{m} g_{j}(x) u_{j}$ and $h(x, u)=h(x)$, this definition reduces to the usual definition of the unobservability distribution [8].

Now let us come back to our algorithm. We need the following technical lemma.

Lemma 4. The subspace of $\mathbb{R}^{n}$ given by

$$
\operatorname{ker}\left[\frac{\partial P_{i}}{\partial x_{j}}\left(x, u, \ldots, u^{\left(k^{*}\right)}, y, \ldots, y^{\left(k^{*}\right)}\right)\right]_{\substack{i=\bar{n}+1, \ldots, n+p \\ j=1, \ldots, n}}
$$

does not depend on $u, \ldots, u^{\left(k^{*}\right)}, y, \ldots, y^{\left(k^{*}\right)}$. 
Proof. By definition of $k^{*}$ we have $p_{k^{*+1}}=s_{k^{*+1}}-s_{k^{*}}=0$. Hence the subspace (45) is also given as

$$
\operatorname{ker}\left[\frac{\partial P_{i}}{\partial x_{j}}\left(x, u, \ldots, u^{\left(k^{*}-1\right)}, y, \ldots, y^{\left(k^{*}-1\right)}\right)\right]_{\substack{i=n_{k} *+1, \ldots n+p \\ j=1 \ldots \ldots n}}
$$

It follows that the subspace (46) does not change if we add to the set $P_{i}, i=n_{k^{*}}+1, \ldots, n+p$, in (46), any function obtained by prolongation of a function in this set and substitution of $\dot{x}=f(x, u)$ in the prolonged function. Now suppose that the subspace given by (45) or by (46) does depend on some variable $u, \ldots, u^{\left(k^{*}-1\right)}, y, \ldots, y^{\left(k^{*}-1\right)}$. Let $r \leq k^{*}-1$ be the highest derivative of $u$ or $y$ such that the subspace (46) depends on $u^{(r)}$ or $y^{(r)}$. Then prolong all the functions $P_{n_{k *+1}}, \ldots, P_{n+p}$ and substitute $\dot{x}=f(x, u)$, and add all these functions to the set $P_{i}, i=n_{k^{*}}+1, \ldots, n+p$, in (46). It follows that the subspace (46) depends non-trivially on $u^{(r+1)}$ or $y^{(r+1)}$, which contradicts the definition of $r$.

It follows from Lemma 4 that (45) defines a distribution $F$ on $\mathbb{R}^{n}$, which is clearly involutive and by assumption of regularity of constant dimension $\bar{n}$. By Frobenius' theorem we can choose local coordinates $\left(x^{1}, x^{2}\right)$, with $\operatorname{dim} x^{1}=\bar{n}$, such that $F=\operatorname{span}\left\{\partial / \partial x^{1}\right\}$. It follows that (35) in such coordinates takes the form

$$
\begin{aligned}
& \dot{x}^{1}-f^{1}\left(x^{1}, x^{2}, u\right)=0, \quad x^{1}=\left(x_{1}, \ldots, x_{\bar{n}}\right) \\
& P_{i}\left(x^{2}, u, \ldots, u^{\left(k^{*}\right)}, y, \ldots, y^{\left(k^{*}\right)}\right)=0, \quad i=\bar{n}+1, \ldots, n+p .
\end{aligned}
$$

Comparing this with (44) and remembering that the equations $y_{i}-h_{i}(x, u)=0, i=1, \ldots, p$, are contained in the equations $P_{\bar{n}+1}=\cdots=P_{n+p}=0$, it can be immediately concluded that $\operatorname{span}\left\{\partial / \partial x^{1}\right\}$ equals $\bar{O}$, the unobservability distribution of (1), since analogously to the case $\bar{n}=0$ (cf. (40a)), we can solve for the variables $x^{2}$ from $s_{k *}\left(=n-\bar{n}=\operatorname{dim} x^{2}\right)$ of the equations $P_{i}=0, i=n_{k}+1, \ldots, n+p$. Therefore we have obtained the following generalization of Theorem 2 .

Theorem 5. Consider the system (1), and let $(\bar{x}, \bar{u}, \bar{y})$ be a regular point for the algorithm. Then (1) can be transformed around $(\bar{x}, \bar{u}, \bar{y})$ into a system of equations

$$
\begin{aligned}
& \dot{x}^{1}=f^{1}\left(x^{1}, x^{2}, u\right), \quad \operatorname{dim} x^{1}=\bar{n}, \\
& x^{2}=\psi\left(u, \ldots, u^{\left(k^{*}-1\right)}, y \ldots, y^{\left(k^{*}-1\right)}\right), \quad \operatorname{dim} x^{2}=n-\bar{n}, \\
& P_{i}^{\mathrm{e}}\left(u, \dot{u}, \ldots, u^{\left(k^{*}\right)}, y, \dot{y}, \ldots, y^{\left(k^{*}\right)}\right)=0, \quad i=1, \ldots, p .
\end{aligned}
$$

Here (48a) are the dynamics of the unobservable part $\left(h(x, u)=h\left(x^{2}, u\right)\right)$, (48b) expresses the remaining state as a function of $u$ and $y$ and their derivatives, and (48c) describes the external behaviour of the system.

Remark 1. The integers $p_{1}, p_{2}, \ldots, p_{k}$ appearing in the algorithm have an immediate interpretation. Define

$$
\kappa_{i}=\#\left\{p_{j} ; p_{j} \geq i\right\}, \quad i=1, \ldots, p .
$$

It is easily checked that

$$
\begin{aligned}
& p_{1} \geq p_{2} \geq \cdots \geq p_{k^{*}}>0, \quad p_{1}+p_{2}+\cdots+p_{k^{*}}=n-\bar{n}, \\
& \kappa_{1} \geq \kappa_{2} \geq \cdots \geq \kappa_{p} \geq 0, \quad \kappa_{1}+\kappa_{2}+\cdots+\kappa_{p}=n-\bar{n} .
\end{aligned}
$$

The integers $\kappa_{1}, \ldots, \kappa_{p}$ can be called the observability indices of the nonlinear system (1) (see also [5] for the case $p=1$ ). Indeed for a linear system (see Remark 2) this definition coincides with the usual one. 
Remark 2. If (1) is a linear system then we have the following frequency-domain version of (2)

$$
\left[\begin{array}{ccc}
s I-A & -B & 0 \\
-C & -D & I
\end{array}\right]\left[\begin{array}{l}
x(s) \\
u(s) \\
y(s)
\end{array}\right]=0, \quad x \in \mathbb{R}^{n}, u \in \mathbb{R}^{m}, y \in \mathbb{R}^{p} .
$$

The specialization of Theorem 2 to this case is as follows. It is well known that if $(C, A)$ is observable then there exists an $(n+p) \times(n+p)$ unimodular matrix $U(s)$ such that

$$
U(s)\left[\begin{array}{c}
s I-A \\
-C
\end{array}\right]=\left[\begin{array}{c}
I_{n} \\
0
\end{array}\right] .
$$

Premultiplication of (51) by $U(s)$ yields an expression of the form (for some polynomial matrices $R_{1}(s)$, $\left.R_{2}(s), P_{1}(s), P_{2}(s)\right)$ :

$$
\left[\begin{array}{ccc}
I & -R_{1}(s) & -R_{2}(s) \\
0 & -P_{1}(s) & P_{2}(s)
\end{array}\right]\left[\begin{array}{l}
x(s) \\
u(s) \\
y(s)
\end{array}\right]=0
$$

so that $x(t)=R_{1}(\mathrm{~d} / \mathrm{d} t) u(t)+R_{2}(\mathrm{~d} / \mathrm{d} t) y(t)$, and $P_{2}(\mathrm{~d} / \mathrm{d} t) y(t)=P_{1}(\mathrm{~d} / \mathrm{d} t) u(t)$. Since $U(s)$ is unimodular, the equations (51) and (53) are equivalent, and our algorithm precisely corresponds to the computation of $U(s)$. Theorem 5 can be interpreted in a similar way. Undoubtedly such a result is known in linear systems theory; however we have not found any explicity statement of it.

Remark 3. The algorithm can be easily extended to implicit (singular) state space systems $F(x, \dot{x}, u)=0$, $y=h(x, u)$. The main difference is that in this case we have to use the implicit function theorem at every step of the algorithm and not, as in the present case, only at the last step to solve for the observable part of the state.

Example. Consider the bilinear system treated in [2],

$$
\begin{aligned}
& \dot{x}_{1}-u x_{2}=: P_{1}(x, \dot{x}, u)=0, \\
& \dot{x}_{2}-u x_{1}=: \dot{P}_{2}(x, \dot{x}, u)=0, \\
& y-x_{2}-u x_{1}=: P_{3}(x, u, y) .
\end{aligned}
$$

Clearly rank $\partial P_{3} / \partial x=\operatorname{rank}(-u,-1)=1$ everywhere, and $\dot{P}_{3}=\dot{y}-\dot{x}_{2}-u \dot{x}_{2}-\dot{u} x_{1}$. Hence at the first step of the algorithm we transform (54) into

$$
\begin{aligned}
& 0=\dot{x}_{1}-u x_{2}=P_{1}(x, \dot{x}, u), \\
& 0=\dot{y}-u x_{1}-u^{2} x_{2}-\dot{u} x_{1}=: P_{2}(x, u, \dot{u}, y, \dot{y}), \\
& 0=y-x_{2}-u x_{1}=P_{3}(x, u, y) .
\end{aligned}
$$

For the second step we notice that

$$
\left[\begin{array}{c}
\frac{\partial P_{2}}{\partial x} \\
\frac{\partial P_{3}}{\partial x}
\end{array}\right]=\left[\begin{array}{cc}
-u-\dot{u} & -u^{2} \\
-u & -1
\end{array}\right]
$$

which has rank 2 for $u+\dot{u}-u^{3} \neq 0$. Excluding this point we define

$$
S_{2}=\dot{P}_{2}-u^{2} \dot{P}_{3}=\ddot{y}-u^{2} \dot{y}-2 u \dot{u} \dot{y}-\left(u+\dot{u}-u^{3}\right) \dot{x}_{1}-\left(\dot{u}+\ddot{u}-3 u^{2} \dot{u}\right) x_{1}
$$


so that $S_{2}$ does not depend on $\dot{x}_{2}$, and $\partial S_{2} / \partial \dot{x}_{1}=-\left(u+\dot{u}-u^{3}\right)$. Then (55) is for $u+\dot{u}-u^{3} \neq 0$ equivalent to

$$
\begin{aligned}
& \dot{y}-u^{2} \dot{y}-2 u \dot{u} \dot{y}-\left(u+\dot{u}-u^{3}\right) u x_{2}-\left(\dot{u}-\ddot{u}-3 u^{2} \dot{u}\right) x_{1}=0, \\
& \dot{y}-u x_{1}-u^{2} x_{2}-\dot{u} x_{1}=0, \\
& \dot{y}-x_{2}-u x_{1}=0 .
\end{aligned}
$$

From the last two equations of (58) we can solve for $x_{1}$ and $x_{2}$ provided $u+\dot{u}-u^{3} \neq 0$. Substitution in the first equation of (58) then gives the higher-order equation describing the external behaviour of the system.

\section{References}

[1] G. Conte, C.M. Moog and A. Perdon, Un theorème sur la représentation entrée-sortie d'un système nonlinéaire, C.R. Acad. Sci. Paris Sér. I 307 (1988) 363-366.

[2] P.E. Crouch and F. Lamnabhi-Lagarrigue, State space realizations of nonlinear systems defined by input-output differential equations, in: A. Bensoussan, J.L. Lions, Eds., Analysis and Optimization of Systems, LNCIS 111 (Springer, Berlin, 1988) $138-149$.

[3] S. Diop, A state elimination procedure for nonlinear systems, preprint (1988); presented at the Nantes Nonlinear Control Conference (June 1988).

[4] M. Fliess, A note on the invertibility of nonlinear input-output systems, Sytems Control Lett. 8 (1986) 147-151.

[5] M. Fliess, Nonlinear control theory and differential algebra, in: C.I. Byrnes, A. Kurzhanski, Eds., Modelling and Adaptive Control, LNCIS 105 (Springer, Berlin, 1988) 134-145.

[6] M.I. Freedman and J.C. Willems, Smooth representations of systems with differentiated inputs, IEEE Trans. Automat. Control 23 (1978) 16-22.

[7] S.T. Glad, Nonlinear state space and input output descriptions using differential polynomials, Preprint (1988); presented at the Nantes Nonlinear Control Conference (June 1988).

[8] A. Isidori, A.J. Krener, C. Gori-Giorgi and S. Monaco, Nonlinear decoupling via feedback: a differential geometric approach, IEEE Trans. Automat. Control 26 (1981) 331-345.

[9] W. Respondek, Transforming a nonlinear control system into a differential equation in the inputs and outputs (in preparation).

[10] W. Respondek and H. Nijmeijer, On local right-invertibility of nonlinear control systems, Control Theory and Adianced Technology 4 (1988) 325-348.

[11] A.J. van der Schaft, On realization of nonlinear systems described by higher-order differential equations. Math. Systems Theory 19 (1987) 239-275; Correction 20 (1987) 305-306.

[12] A.J, van der Schaft, Transformations of nonlinear systems under external equivalence, Memo 700, University of Twente (March 1988); presented at the Nantes Nonlinear Control Conference (June 1988).

[13] A.J. van der Schaft, System theoretic descriptions of physical systems, CWI Tract 3 (CWI. Amsterdam, 1984).

[14] J.M. Schumacher, Transformations of linear systems under external equivalence, Linear Algebra Appl. 102 (1988) 1-33.

[15] E.D. Sontag, Bilinear realizability is equivalent to existence of a singular affine differential I/O equation, Systems Control Lett. 11 (1988) 181-187.

[16] J.C. Willems, Input-output and state-space representations of finite-dimensional linear time-invariant systems, Linear Algebra Appl 50 (1983) $581-608$.

[17] J.C. Willems, From time series to linear system - Part I. Finite dimensional linear time invariant systems, Automatica 22 (1986) $561-580$ 